'Departamento de Cirugía Digestiva. Hospital Clínico de la Pontificia Universidad Católica de Chile. Santiago, Chile.

Trabajo no recibió financiamiento.

Los autores declaran no tener conflictos de interés.

Recibido el 18 de marzo de 2018, aceptado el 18 de mayo de 2018 .

Correspondencia a: Dr. Eduardo Briceño Diagonal Paraguay 362, Piso 4, Santiago, Chile. ebriceno@puc.cl

\section{Fascitis necrotizante de la pared abdominal como presentación infrecuente de una hernia de Garengeot: Caso clínico}

\author{
EDUARDO BRICEÑO ${ }^{1}$, ROCÍO JARA ${ }^{1}$
}

\section{Necrotizing fasciitis caused by a Garengeot's hernia. Case report}

Garengeot's hernia corresponds to the presence of the appendix within a femoral hernia, associated or not with acute appendicitis. The diagnosis of this uncommon situation is usually done during surgery. Furthermore, the clinical presentation as necrotizing fasciitis is a rare condition. We report a 54 years old obese hypertensive woman with rheumatoid arthritis of 40 years of evolution treated with methotrexate and prednisone. She consulted for pain and erythema in the right inguinal region. Laboratory revealed leukocytosis and an elevated $C$-reactive Protein. Suspecting a cellulitis, the patient was admitted for antimicrobial therapy. A pelvic magnetic resonance imaging showed a perforated acute appendicitis in an inguinal hernia with extensive pelvic cellulitis associated with signs of fasciitis. At surgery, an extensive groin and pubic fasciitis was evident, with a necrotic and perforated appendix within a femoral hernia. Surgical debridement, open appendectomy, and femoral hernioplasty without mesh were carried out. Vacuum-assisted closure was installed in the coverage defect. Three surgical debridement procedures were required for the closure of the wound. Two weeks after the first surgical procedure, the patient was discharged in good condition. During the follow-up, she evolved with a surgical wound dehiscence, which was managed with wound dressings until closure.

(Rev Med Chile 2018; 146: 660-664)

Key words: Appendicitis; Fasciitis, Necrotizing; Hernia, Femoral; Negative-Pressure Wound Therapy.

\section{L} a hernia de Garengeot corresponde a la presencia del apéndice cecal dentro de una hernia femoral, asociado o no a una apendicitis aguda ${ }^{1}$. Es una entidad clínica infrecuente, lo que determina una baja sospecha clínica y genera que en la mayoría de los casos el diagnóstico se realice en forma intraoperatoria al intervenir a un paciente con una hernia femoral atascada ${ }^{2}$. La presentación como fascitis necrotizante de la pared abdominal es anecdótica ${ }^{3,4}$. Se presenta el caso de una mujer de 54 años que debutó con una fascitis de la pared abdominal secundario a una apendicitis aguda dentro de una hernia femoral. Como tratamiento se realizó un extenso aseo quirúrgico inicial más apendicectomía, herniorrafia e instalación de sistema $\mathrm{VAC}^{\circledR}$.

\section{Caso clínico}

Mujer de 54 años, obesa, antecedente de hipertensión arterial y artritis reumatoidea juvenil de larga data, en tratamiento con metrotrexato $10 \mathrm{mg}$ semanales y prednisona $10 \mathrm{mg}$ diarios. Consultó en servicio de urgencia por 3 días de dolor inguinal 
derecho asociado a eritema. Al ingreso se constató taquicárdica (110/min) y subfebril. En el exámen físico (Figura 1) se evidenció una extensa celulitis de la región inguinal derecha con extensión hacia la raíz del muslo derecho y zona púbica, con un área de mayor induración y sensibilidad en relación a la región inguinal. En sus exámenes destaca una PCR de 39 y leucocitosis de 28.000, con $98 \%$ neutrofilos, sin otras alteraciones.

Se realizó una resonancia nuclear magnética (RNM) pélvica (Figura 2) que demostró un apéndice necrótico y perforado dentro de un saco herniario asociado a extensos signos de celulitis y fascitis de la pared. Fue reanimada con cristaloides y se inició antibioticoterapia empírica con ampicilina-sulbactam.

Se realizó un aseo quirúrgico (Figura 3 ) evidenciando tejidos fascíticos de mal olor y drenando una colección purulenta en relación al triángulo femoral. Se resecaron los tejidos desvitalizados hasta obtener márgenes sanos. Se enviaron muestras de tejidos y de líquido purulento para cultivo. Se evidenció un apéndice cecal necrótico y perforado dentro de un saco herniario femoral, encontrando una base apendicular sana y adecuada para su ligadura y descartando la extensión intrabdominal de la infección, no siendo necesario ingresar a la cavidad abdominal. Se resecó el saco herniario y se realizó una apendicectomia clásica.

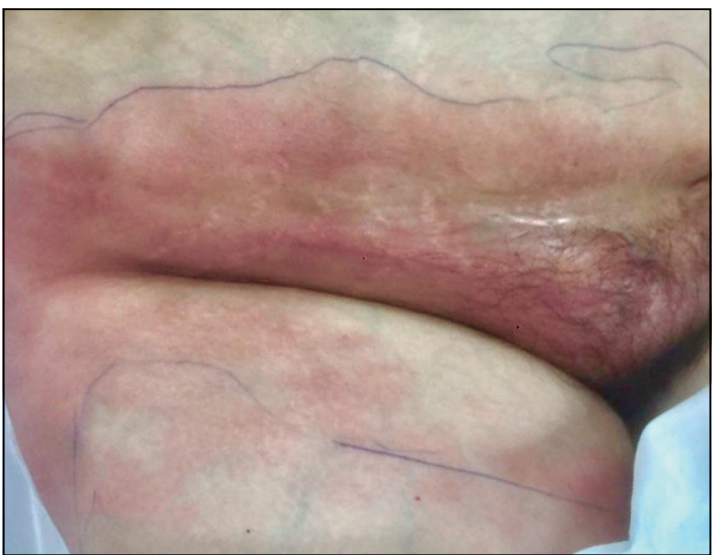

Figura 1. Extensa celulitis inguinal derecha, con extensión a pared abdominal inferior, zona púbica y raíz del muslo derecho. En azul se delimitaron cambios inflamatorios locales. Se observa la progresión de la signología clínica a las 8 h de evolución.
Se identificaron los ligamentos inguinal y pectíneo y se realizó una herniorrafia femoral mediante técnica infrainguinal.

Se completó el debridamiento y se lavó profusamente la zona con suero fisiológico y solución de clorhexidina. Para el defecto de cobertura se utilizó un sistema aspirativo continuo $\left(\mathrm{VAC}^{\circledR}\right)$.

Los cultivos fueron positivos para Streptococcus constellatus. La paciente evolucionó en forma satisfactoria, con normalización de los parámetros inflamatorios y asintomática del punto de vista digestivo. A las $48 \mathrm{~h}$ de tratamiento antibiótico y de terapia $\mathrm{VAC}^{\circledR}$ fue sometida a un nuevo aseo quirúrgico donde se descartó la progresión de la infección y se evidenció una clara mejoría de las condiciones locales (Figura 4).
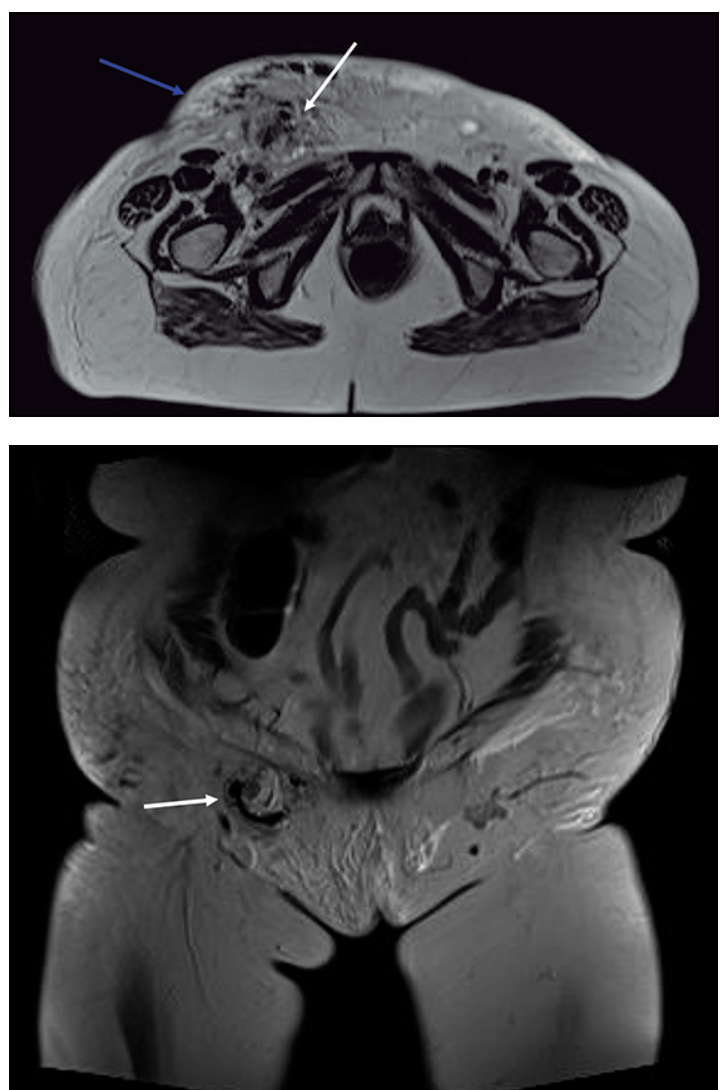

Figura 2. Imágenes axial (a) y coronal (b) de Resonancia Nuclear Magnética de pelvis que demuestran apéndice cecal necrótico con signos de perforación en el interior de un saco herniario inguinal (flecha blanca) y extensos cambios inflamatorios de los tejidos (flecha azul). 

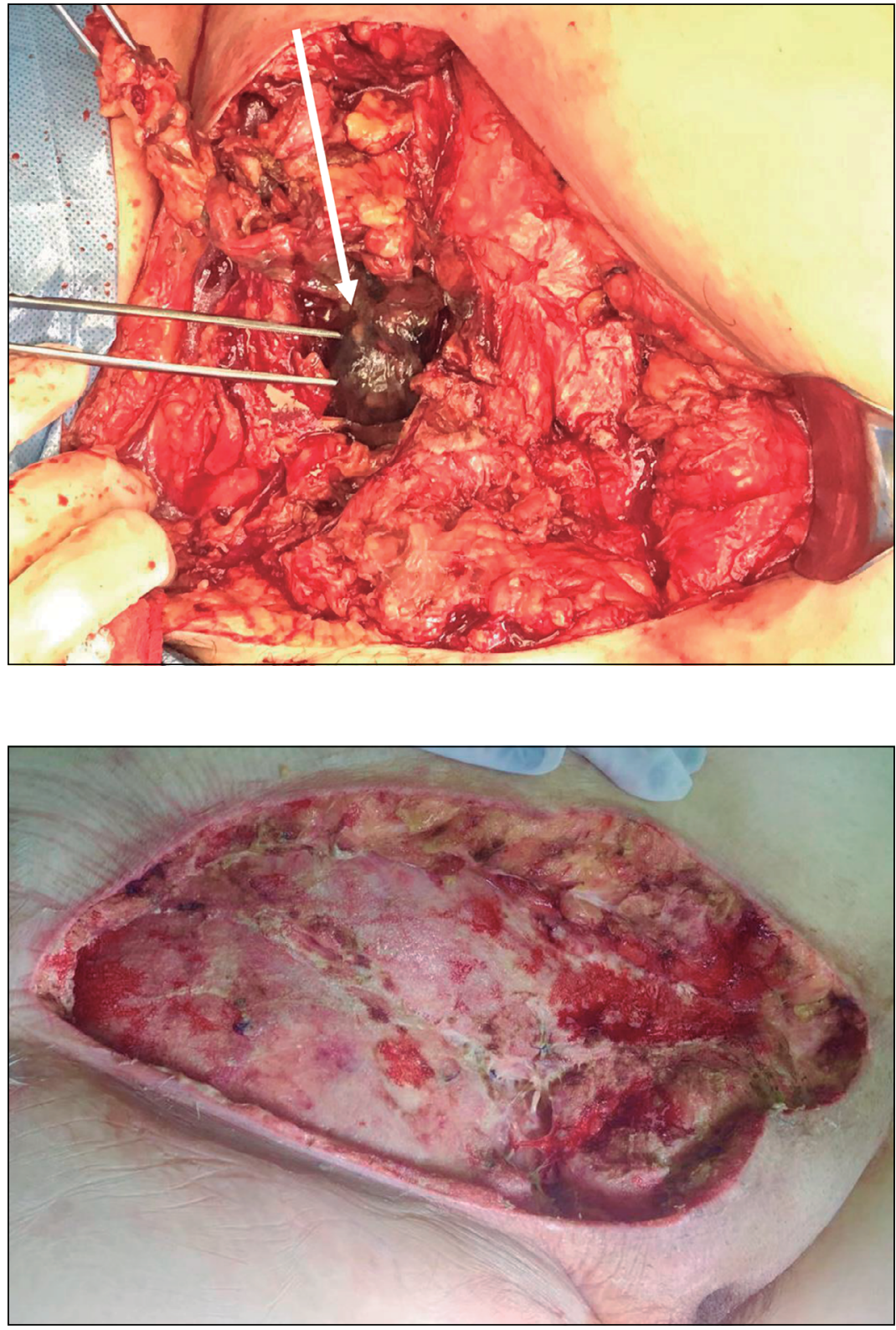

Figura 3. Imagen intraoperatoria donde se observa la zona inguinal y crural derecha posterior a aseo quirúrgico. La flecha muestra el apéndice necrótico.

Figura 4. Imagen intraoperatoria de la misma zona inguinal y crural derecha con su defecto de cobertura, posterior a un segundo aseo quirúrgico más instalación de sistema $V A C^{\circledR}$.

Luego de 2 nuevos aseos quirúrgicos para definir los bordes de la herida y con cultivos de tejido negativo, fue posible el cierre definitivo de ésta. A las dos semanas de su ingreso, la paciente fue dada de alta en buenas condiciones. En el seguimiento se constató la dehiscencia parcial de la herida operatoria, la que fue manejada con curaciones hasta su cierre.

\section{Discusión}

Las hernias femorales corresponden al 4\% de las hernias de la región inguinal y son más frecuentes en mujeres que hombres (razón 4:1-6:1). Son menos comunes que las hernias inguinales en ambos sexos ${ }^{1}$, pero presentan un alto riesgo de estrangulación $(5-20 \%)^{5,6}$. 
La hernia de Garengeot corresponde a la presencia del apéndice cecal dentro de un saco herniario femoral y fue descrita por primera vez en 1731 por el cirujano francés Rene Jacques Croissant de Garengeot (1688-1759) ${ }^{1}$.

Representa un hallazgo inusual e incidental que ocurre aproximadamente en $1 \%$ de todas las hernias femorales, siendo la incidencia de apendicitis incluso más rara $(0,08-0,13 \%$ de los casos).

En contraste a la hernia de Amyand (apendicitis dentro de una hernia inguinal), presenta una significativa predisposición por mujeres postmenopáusicas ${ }^{2}$.

Esta fuerte prevalencia ha sido atribuida a los cambios del anillo femoral durante el desarrollo y el embarazo. Otros factores de riesgo incluyen la presión intraabdominal incrementada, el envejecimiento, el tabaquismo, desórdenes musculares y del tejido conectivo, además de un ciego de implantación baja y un apéndice pélvico que facilitarían su entrada al canal femoral ${ }^{3,7}$.

La fisiopatología estaría relacionada a la anatomía del canal femoral, con sus bordes rígidos y estrechos, que determinarían que el estrangulamiento y la isquemia del apéndice puedan producirse rápidamente, lo que también podría explicar la ausencia de apendicolitos como causa de la apendicitis en estos casos. Estas mismas características anatómicas del anillo femoral prevendrían la diseminación intraperitoneal de la infección, siendo muy infrecuentes los síntomas de peritonitis, ${ }^{4,7}$.

Se presenta habitualmente como una hernia inguinal atascada o estrangulada y el diagnóstico es mayormente realizado en forma intraoperatoria. Esta presentación clínica atípica es debida a la ausencia de síntomas abdominales y a sus hallazgos radiológicos inespecíficos ${ }^{2,4}$.

Kalles et $\mathrm{al}^{4}$ en su revisión, analizaron 36 pacientes en 31 artículos. Todos presentaron una masa o tumefacción dolorosa en la región inguinal, habitualmente derecha (97\%). Sólo 14\% reportó síntomas digestivos como náuseas, vómitos o cambios en el hábito intestinal. El 97\% presentaba al examen físico una masa o tumefacción inguinal dolorosa, asociado a eritema en 56\% de los casos. La mayoría presentaba leucocitosis y PCR elevada. Entre 14 y 31\% presentaba un estudio imagenológico preoperatorio, siendo la Tomografía Axial Computarizada (TAC) la de mejor rendimiento, con $44 \%$ de diagnósticos positivos para apendicitis dentro de la hernia femoral.
Finalmente, sólo en 14\% de los casos se hizo un diagnóstico preoperatorio de hernia de Garengeot, que corresponde a aquellos pacientes que tuvieron un TAC positivo. Pese a este resultado, el TAC es el estudio de imágenes inicial recomendado para la evaluación de un paciente con una masa o signos inflamatorios inguinales dado su excelente rendimiento y alta capacidad diagnóstica ${ }^{4}$. Sin embargo, es importante recalcar que la conducta frente a una sospecha de hernia atascada o estrangulada es la exploración quirúrgica, la que no debe retrasarse por la espera de un estudio radiológico ${ }^{2}$.

Sólo un paciente $(2,7 \%)$ presentó una fascitis necrotizante de la región inguinal, en el que se describe un divertículo apendicular perforado como la causa del proceso infeccioso ${ }^{3,4}$, siendo el caso que estamos presentando uno de los pocos reportes descritos en la literatura de una hernia de Garengeot asociada a una fascitis necrotizante de la pared abdominal ${ }^{4}$.

Dado la ausencia de síntomas y signos abdominales de apendicitis, la hernia de Garengeot debería ser sospechada en los pacientes que se presentan con una masa dolorosa inguinal derecha. Los diagnósticos diferenciales a considerar además de una hernia complicada son: anexitis en el contexto de una hernia inguinal, ectasia de la vena safena magna, lipomas, tumores de partes blandas, adenopatias reactivas, linfomas y abscesos hipostáticos en procesos retroperitoneales ${ }^{7,8}$.

Por su rareza existen múltiples reportes de casos clínicos y escasas series publicadas, lo que dificulta obtener un consenso respecto a su tratamiento ${ }^{2,6}$. Distintas estrategias de tratamiento $\mathrm{y}$ abordajes quirúrgicos se han descrito, las que dependen de la condición del paciente, de sus comorbilidades y del criterio de cada cirujano ${ }^{6}$. La vía de abordaje más frecuente es la infrainguinal ${ }^{4,9}$ realizando por este mismo acceso la apendicectomía y la reparación herniaria, mayormente sin malla dado el ambiente infeccioso local. Sin embargo, hay algunos casos clínicos de reparaciones con malla por vía abierta o laparoscópica en pacientes con apéndices no perforados que no reportan complicaciones infecciosas locales s,6,7,8,10 $^{\text {. También }}$ se describe el abordaje exclusivo por laparotomía o combinado con una incisión inguinal ${ }^{4,6}$.

La aproximación por laparoscopia es una atractiva y poco invasiva opción para realizar la apendicectomía y reparar el defecto herniario, permitiendo además explorar la cavidad abdo- 
minal ${ }^{6,8,11,12}$, pero no es planteable como vía única de tratamiento en un paciente con sospecha de infección a nivel inguinal que requiere de un aseo quirúrgico local. Frente a esta situación, se emplean abordajes combinados mediante apendicectomía por laparoscopia e incisión inguinal para aseo y herniorrafia ${ }^{12}$.

En nuestro caso el abordaje infrainguinal permitió una adecuada visualización de la base apendicular y una reparación herniaria satisfactoria sin necesidad de malla.

El diagnóstico diferencial entre celulitis y fascitis necrotizante inicial es clínicamente difícil. Debe hacer sospechar esta última, la rápida progresión de los síntomas, crepitación de tejidos blandos y el compromiso sistémico. Dada la elevada morbilidad y mortalidad de esta entidad es necesario el diagnóstico precoz. El tratamiento es mediante un debridamiento extenso de los tejidos desvitalizados, distinto al de la celulitis, en el que se realiza principalmente terapia con antibióticos ${ }^{13,14}$.

Respecto a la terapia con $\mathrm{VAC}^{\circledR}$, se ha descrito y demostrado su utilidad para el tratamiento de los grandes defectos de cobertura cutánea y en especial en un contexto de infección ${ }^{13}$ y fue un complemento fundamental para la rápida y adecuada recuperación de nuestra paciente.

\section{Referencias}

1. Akopian G, Alexander M. De Garengeot hernia: appendicitis within a femoral hernia. Am Surg 2005; 71 (6): 526-7.

2. Rajan S, Girn H, Ainslie W. Inflamed appendix in a femoral hernial sac: de Garengeot's hernia. Hernia 2009; 13 (5): 551-3.

3. Georgiou G, Bali C, Theodorou S, Zioga A, Fatouros M. Appendiceal diverticulitis in a femoral hernia causing necrotizing fasciitis of the right inguinal region: report of a unique case. Hernia 2013; 17: 125-8.

4. Kalles V, Mekras A, Mekras D, Papapanagiotou I, Al-Harethee W, Sotiropoulos G, et al. De Garengeot's hernia: a comprehensive review. Hernia 2013; 17: 17782.

5. Mizumoto R, Hendahewa R, Premaratne G. De Garengeot hernia- Use of a novel surgical approach and literature review. Int J Surg Case Rep 2016; 19: 127-30.

6. Kagan Coskun A, Kilbas Z, Yigit T, Simsek A, Harlak A. De Garengeot's hernia: the importance of early diagnosis and its complications. Hernia 2012; 16: 731-3.

7. Sharma H, Jha P, Shekhawat N, Memon B, Memon M. De Garengeot hernia: an analysis of our experience. Hernia 2007; 11: 235-8.

8. Comman A, Gaetzschmann P, Hanner T, Behrend M. DeGarengeot hernia: transabdominal preperitoneal hernia repair and appendectomy. JSLS 2007; 11 (4): 496-501.

9. Hachisuka T. Femoral hernia repair. Surg Clin North Am 2003; 83 (5): 1189-205.

10. Ebisawa K, Yamazaki S, Kimura Y, Kashio M, Kurito K, Yasumuro S, et al Acute appendicitis in an incarcerated femoral hernia: a case of de Garengeot hernia. Case Rep Gastroenterol 2009; 3: 313-7.

11. Díaz R, Rodríguez F, Bustos A, Alfaro J. Cirugía laparoscópica de una hernia de Garengeot: presentación poco frecuente de una hernia crural. Rev Chil Cir 2016; 68 (6): 446-8.

12. Thomas B, Thomas M, McVay B, Chivate J. De Garengeot hernia. JSLS 2009; 13: 455-7.

13. Beerle C, Gelpke H, Breitenstein S, Staerkle RF. Complicated acute appendicitis presenting as a rapidly progressive soft tissue infection of the abdominal wall: a case report. Journal of Medical Case Reports 2016; 10: 331.

14. Stevens D, Bryant A. Necrotizing Soft-Tissue Infections. N Engl J Med 2017; 377: 2253-65. 